\title{
Massive pulmonary hemorrhage and severe retinopathy of prematurity in very low birth weight infants
}

\author{
Na Young Lee ${ }^{1}$, Hyun Young Lee ${ }^{1}$, Shin Hae Park ${ }^{2}$, In Kyung Sung ${ }^{1}$, YoungAh Youn ${ }^{1}$ \\ Department of ${ }^{1}$ Pediatrics and ${ }^{2}$ Ophthalmology, Seoul St. Mary's Hospital, College of Medicine, The Catholic University \\ of Korea, Seoul, Republic of Korea. E-mail: lea732@hanmail.net \\ Received: 5th May 2017, Accepted: 30th July 2017
}

SUMMARY: Lee NY, Lee HY, Park SH, Sung IK, Youn YA. Massive pulmonary hemorrhage and severe retinopathy of prematurity in very low birth weight infants. Turk J Pediatr 2017; 59: 636-641.

To examine several clinical factors for association with the development of greater than stage 2 of severe retinopathy of prematurity (ROP) and those who required laser treatment in very low birth weight infants (VLBWI). Between Dec 2013 and Jan 2015, the clinical data of 191 VLBWI admitted, were retrospectively analyzed for ROP. Of a total 191 VLBWI, $18.8 \%(62 / 191)$ infants had severe ROP and $5.8 \%(11 / 191)$ neonates had laser treatment for progressive ROP. ROP neonates were significantly lower gestational age and had higher incidence of morbidities. Among the VLBWI with ROP $(n=62)$, severe ROP group also had significantly lower birth weight and higher incidence of hospital morbidities. Massive pulmonary hemorrhage was consistently associated with severe ROP in our logistic regression analysis. Severe ROP group was significantly related to increased hospital morbidities and mortality. Massive pulmonary hemorrhage may pose to the development of severe ROP.

Key words: laser treatment, risk factor, pulmonary hemorrhage, retinopathy of prematurity.

Over the past two decades, there have been progressive advances in neonatal practice along with antenatal care, which has led to increased survival rate of very low and extremely premature infants. Increased survival in this population has resulted in a higher risk of developing complications like retinopathy of prematurity (ROP). In developed countries, higher complications of preterm birth are the leading causes of mortality. Therefore, assessing high risk factors and minimizing morbidities are important in our clinical practice. ROP remains one of the leading causes of preventable childhood blindness. ROP pathogenesis is multifactorial and known to be associated with lower gestational age and birth weight and further associated with uncontrolled use of oxygen therapy. Some studies have suggested various postnatal factors, such as episodes and frequency of apnea, sepsis, a history of surfactant treatment, multiple transfusions, anemia, or multiple births, as risk factors of ROP. ${ }^{1-4}$ Gotsch et al. ${ }^{5}$ reported that ROP is a vasoproliferative disease, and an antiangiogenic state in mothers with preeclampsia might slow vasoproliferation which may protect infants from ROP. Furthermore, ROP rates are also reported differently perhaps related to geographic areas, genetics differences, or environmental susceptibility. ${ }^{6-11}$

We evaluated several clinical factors for association with the development of greater than stage 2 as severe ROP and those who required laser treatment. The goal of this study was to identify the statistically independent risk factors for ROP development in very low birth weight infants (VLBWI). Identification of these risk factors may help more stable prediction of high-risk infants.

\section{Material and Methods}

The VLBWI admitted to the neonatal intensive care unit at Seoul St. Mary's Hospital during 2013-2015 were analyzed to determine clinical factors influencing the development of severe 
ROP. We analyzed demographic and clinical data abstracted from the medical records of 191 VLBWI who were examined for ROP during this period. Data on infant characteristics and outcomes for this study was extracted. The infants who later need laser operation were compared in relation to infants without laser operation. Our study included VLBWI screened for ROP with birth weight $<1500 \mathrm{~g}$ and gestational age $<32$ weeks at birth during this study period. The initial ophthalmologic examination was performed between the 4th and 6 th week after birth. They were examined until resolution or until effective stabilization of retinopathy was achieved after treatment. The study was approved by the Ethics Committee of Seoul St. Mary's Hospital, the Catholic University of Seoul, Korea (KC16OISI0645, 201607). Since it was a retrospective study, informed consent of participants was not required.

\section{Definitions}

By definition, ROP was defined with the classified according to the International Classification of Diseases for ROP. Severe ROP was defined as greater than stage 2 or those who required laser treatment in this study. ${ }^{12}$ Massive pulmonary hemorrhage was defined as pulmonary hemorrhage which affects vital signs as manifested by cardiovascular collapse or acute respiratory failure. Pulmonary hypertension is defined by the need to use nitrioxide or sildenafil or iloprost $\leq 1$ week of life. Bronchopulmonary dysplasia (BPD) was diagnosed if oxygen use exceeding $0.21 \%$ was still needed at a corrected gestational age of 36 weeks. Necrotizing enterocolitis (NEC) was defined as grade II or higher using Bell's classification. Intraventricular hemorrhage $(\mathrm{IVH})>$ grade II was defined as active bleeding in the ventricles, and the grade designation was based on Drs. Papile's classification criteria ${ }^{13}$.

\section{Statistical Analysis}

Continuous variables were compared using Student's t-test and are expressed as the means \pm of the standard deviations. Discrete variables were compared using a $\chi^{2}$ test or Fisher's exact test and are expressed as percentages. All of the analyses were two-tailed, and clinical significance was defined as a $\mathrm{p}$ value lower than 0.05 . To seek any confounding risk factors for severe ROP, we used a multivariate logistic regression model. Odds ratios (ORs) and $95 \%$ confidence intervals (CI) were calculated using a multivariate statistical model that included the following predictors related to severe ROP with a stepwise logistic regression analysis: gestational age, birth weight, pulmonary hemorrhage, and pulmonary hypertension. All the statistical analyses were performed with the Statistical Package for the Social Sciences (SPSS), version 15.0 (SPSS-PC Inc., Chicago, IL, USA).

\section{Results}

Of the 191 neonates with $\leq 1500$ g birth weight, admitted to the neonatal intensive care unit between December 2013 to January 2015 who survived until the initial ophthalmologic examination performed between the 4 th and 6th week after birth, $93.7 \%$ (141/191) survived. $32.4 \%(62 / 191)$ infants had ROP among the VLBWI and $18.8 \%(62 / 191)$ of infants had severe ROP. Among these, 11 neonates had laser treatment for severe and progressive ROP. Infants with ROP were of significantly lower gestational age $(27.3 \pm 1.9$ weeks vs and $28.1 \pm 2.7$ weeks, $\mathrm{p}=0.037)$ and had a higher incidence of maternal chorioamnionitis, use of surfactant $\geq 2$ times and neonatal seizure when compared to those who did not have ROP $(p<0.05)$. As in morbidities, infants who developed ROP had significantly more sepsis, patent ductus arteriosus (PDA) ligation, $\mathrm{BPD} \geq$ moderate and periventricular leukomalacia (PVL). Also, they had longer days to reach full feeding, total parental nutrition (TPN) and mechanical ventilation duration and hospitalized days $(\mathrm{p}<0.001)$. Mortality was also significantly higher in VLBWI with ROP (Table I).

Among the VLBWI with ROP $(n=62)$, the severe ROP group had significantly lower birth weight $(978.9 \pm 226.9 \mathrm{~g}$ vs 1095.9 $\pm 197.8 \mathrm{~g}, \mathrm{p}=0.039$ ), higher incidence of maternal chorioamnionitis, use of surfactant $\geq$ 2 times and massive pulmonary hemorrhage when compared to those with ROP $(p<0.05)$. As in morbidities, severe ROP group had significantly more IVH $\geq$ grade II, sepsis, longer TPN and mechanical ventilation duration and hospitalized days $(p<0.05)$. The ROP laser 
Table I. Clinical Characteristics of VLBWIs $(n=191)$

\begin{tabular}{llll}
\hline & No ROP $(\mathrm{n}=129)$ & ROP $(\mathrm{n}=62)$ & P-value \\
\hline Gestational age, week & $28.1 \pm 2.7$ & $27.3 \pm 1.9$ & $0.037^{*}$ \\
Birth weight, g & $1075.3 \pm 294.2$ & $1027.9 \pm 221.3$ & 0.264 \\
Male, n (\%) & $9(34.6)$ & $19(52.8)$ & 0.200 \\
Antenatal steroid use & $120(93.0)$ & $62(100)$ & 0.826 \\
Maternal chorioamnionitis & $19(28.4)$ & $46(43.4)$ & $0.047^{*}$ \\
Resuscitation at delivery\# & $121(94.5)$ & $57(96.6)$ & 0.537 \\
RDS & $117(91.4)$ & $59(95.2)$ & 0.354 \\
Surfactant $>2$ times & $45(34.8)$ & $26(40.0)$ & $0.010^{*}$ \\
Pneumothorax & $20(15.5)$ & $5(8.1)$ & 0.153 \\
Massive pulmonary hemorrhage & $24(18.6)$ & $16(25.8)$ & 0.256 \\
Pulmonary hypertension $\dagger$ & $19(14.7)$ & $10(16.1)$ & 0.801 \\
Neonatal seizure & $57(44.2)$ & $42(67.7)$ & $0.002^{*}$ \\
IVH $>$ grade II & $34(29.8)$ & $21(33.9)$ & 0.580 \\
Sepsis & $43(33.3)$ & $32(51.6)$ & $0.015^{*}$ \\
PDA ligation & $14(10.9)$ & $14(22.6)$ & $0.032^{*}$ \\
NEC OP & $12(9.8)$ & $11(17.7)$ & 0.120 \\
PRC transfusion & $118(91.5)$ & $61(98.4)$ & 0.065 \\
BPD $\geq$ moderate & $65(50.4)$ & $54(87.1)$ & $<0.001^{*}$ \\
PVL & $38(33.3)$ & $34(55.7)$ & $0.004^{*}$ \\
Full feeding reached, day & $28.5 \pm 24.2$ & $58.1 \pm 34.1$ & $<0.001^{*}$ \\
TPN duration* & $28.5 \pm 24.2$ & $58.1 \pm 34.1$ & $<0.001^{*}$ \\
Mechanical ventilation, days & $15.2 \pm 21.6$ & $39.6 \pm 38.2$ & $<0.001^{*}$ \\
Hospitalized days & $30.1 \pm 2.6$ & $37.6 \pm 4.7$ & $<0.001^{*}$ \\
Mortality & $5(3.9)$ & $7(11.3)$ & $<0.001^{*}$ \\
\hline
\end{tabular}

$* \mathrm{P}<0.05$

\#Resuscitation included oxygen use or positive pressure ventilation or intubation

†Use of nitrioxide, sildenafil or iloprost within 1 week of birth

Abbreviations: VLBWI, very low birth weight infants; ROP, retinopathy of prematurity; g, gram body weight; RDS, respiratory distress syndrome; IVH, intraventricular hemorrhage; PDA, patent ductus arteriosus, NEC; necrotizing enterocolitis; BPD, bronchopulmonary dysplasia; PVL, periventricular leukomalacia; TPN, total parental nutrition

treatment was performed in 11 infants $(5.8 \%)$ in the severe ROP group and the mortality rate was significantly higher in VLBWI with severe ROP; all mortality $(n=7)$ was found in the severe ROP group (Table II). In order to seek any possible risk factors in relation to severe ROP, we performed a multivariable logistic regression analysis to find any confounding factors in relation to severe ROP. We included gestational age, birth weight, massive pulmonary hemorrhage and pulmonary hypertension in this analysis. Massive pulmonary hemorrhage was consistently associated with severe ROP with a 7-fold increased odds ratio (OR: 6.584, 95\% CI: 1.29-33.6) (Table III).

\section{Discussion}

In our retrospective study, we evaluated 191 VLBWI who were admitted to our neonatal intensive care unit during our study period. The incidence of severe ROP among VLBWI was $18.8 \%$ which was similar to other reported studies; Thomas et al. ${ }^{14}$ found that $12.7 \%$ infants developed severe ROP. Until now, lower gestational age and duration of ventilation were known to be strongly associated with severity of ROP. Our study agrees with the two most important risk factors for ROP as many studies on different population and countries have manifested.7, 15-17 
Table II. Clinical Characteristics of VLBWIs with ROP $(n=62)$.

\begin{tabular}{llll}
\hline & ROP $(\mathrm{n}=26)$ & Severe ROP $(\mathrm{n}=36)$ & P-value \\
\hline Gestational age, week & $27.5 \pm 1.7$ & $27.1 \pm 2.0$ & 0.254 \\
Birth weight, g & $1095.9 \pm 197.8$ & $978.9 \pm 226.9$ & $0.039^{*}$ \\
Male, n (\%) & $9(34.6)$ & $19(52.8)$ & 0.200 \\
Antenatal steroid use & $26(100)$ & $36(100)$ & 1.000 \\
Maternal chorioamnionitis & $19(28.4)$ & $46(43.4)$ & $0.047^{*}$ \\
Resuscitation at delivery* & $25(96.2)$ & $32(97.0)$ & 0.963 \\
RDS & $35(97.2)$ & $24(92.3)$ & 0.374 \\
Surfactant $>2$ times & $7(19.4)$ & $19(73.0)$ & $0.005^{*}$ \\
Pneumothorax & $1(3.8)$ & $4(11.1)$ & 0.300 \\
Massive pulmonary hemorrhage & $14(38.9)$ & $2(7.7)$ & $0.006^{*}$ \\
Pulmonary hypertension $\dagger$ & $7(19.4)$ & $3(11.5)$ & 0.404 \\
Neonatal seizure & $17(65.4)$ & $25(69.4)$ & 0.738 \\
IVH > grade II & $4(14.8)$ & $37(43.5)$ & $0.007^{*}$ \\
Sepsis & $22(61.1)$ & $10(38.5)$ & 0.078 \\
PDA ligation & $7(19.4)$ & $7(26.9)$ & 0.487 \\
NEC OP & $3(11.5)$ & $8(22.2)$ & 0.277 \\
PRC transfusion & $25(96.2)$ & $36(100.0)$ & 0.236 \\
ROP laser Tx & $0(0)$ & $11(30.5)$ & $<0.001^{*}$ \\
BPD $\geq$ moderate & $21(80.8)$ & $33(91.7)$ & 0.207 \\
PVL & $11(44.0)$ & $23(63.9)$ & 0.124 \\
Full feeding reached, day & $41.92 \pm 18.5$ & $69.8 \pm 38.0$ & 0.067 \\
TPN duration, day & $41.9 \pm 18.5$ & $69.8 \pm 37.9$ & $<0.001^{*}$ \\
Mechanical ventilation, days & $19.1 \pm 17.2$ & $54.2 \pm 42.6$ & $<0.001^{*}$ \\
Hospitalized days & $64.3 \pm 18.0$ & $94.6 \pm 42.9$ & $0.017^{*}$ \\
Mortality & $0.0(0)$ & $7.0(19.4)$ & \\
\hline
\end{tabular}

* $\mathrm{P}<0.05$

\#Resuscitation included oxygen use or positive pressure ventilation or intubation

†Use of nitrioxide, sildenafil or iloprost within 1 week of birth

Abbreviations: VLBWI, very low birth weight infants; ROP, retinopathy of prematurity; g, gram body weight; RDS, respiratory distress syndrome; IVH, intraventricular hemorrhage; PDA, patent ductus arteriosus, NEC; necrotizing enterocolitis; PRC, packed red cell; BPD, bronchopulmonary dysplasia; PVL, periventricular leukomalacia; TPN, total parental nutrition

Risk factors for ROP are all inter-related with each other. As numerous previous studies have evaluated, our infants with ROP had higher incidence of maternal chorioamnionitis and more unstable clinical course along with longer mechanical ventilation duration and hospitalized days. Further identified risk factors for severe ROP included inflammation associated mother's condition such as maternal chorioamnionitis and initially low compliant lung condition for use of surfactant $\geq 2$ times and massive pulmonary hemorrhage were found more in the severe ROP group $(p<0.05)$. As in morbidities, the severe ROP group had significantly more $\mathrm{IVH} \geq$ grade II $^{18}$, sepsis ${ }^{18}$, longer TPN and mechanical ventilation duration ${ }^{18}$ and hospitalized days, but was not related to previously reported risk factor of blood transfusions. ${ }^{19}$ The longer hospitalization was also shown to be a risk factor for ROP, expressing a more complicated respiratory course in VLBWI who require more interventions such as surfactant, high frequency ventilator (HFOV) and oxygen therapy and often develop BPD and ROP.20

Massive pulmonary hemorrhage was the consistently significant risk factor for severe 
Table III. Risk Factors for Severe ROP in Multiple Logistic Regression Analysis.\#

\begin{tabular}{llll}
\hline & OR & P-value & $95 \%$ CI \\
\hline Gestational age, week & 0.961 & 0.823 & $0.678-1.363$ \\
Birth weight, g & 0.998 & 0.247 & $0.995-1.002$ \\
Pulmonary hemorrhage & 6.584 & 0.023 & $1.292-33.557$ \\
Pulmonary hypertension* & 1.453 & 0.660 & $0.255-7.645$ \\
\hline
\end{tabular}

\# Adjusted for gestational age, birth weight, pulmonary hemorrhage, pulmonary hypertension

* Use of nitrioxide or sildenafil or iloprost within 1 week of birth

Abbreviations: ROP, retinopathy of prematurity

ROP in our logistic regression analysis. Pulmonary hemorrhage is thought to occur due to a more rapid drop in pulmonary vascular resistance after surfactant replacement, which can exacerbate lung congestion with respiratory distress syndrome, leading to pulmonary hemorrhage. ${ }^{21}$ Improvement of respiratory function would decrease the higher and longer need of oxygen therapy, longer mechanical ventilation and secondarily the development of severe ROP. Further, we hypothesized that massive pulmonary hemorrhage may stimulate more of angiogenic state which may be more prone to the occurrence of severe ROP as opposed to the study by Gotsch et al. ${ }^{5}$ that some antiangiogenic factor produced by preeclamptic mothers protect from ROP.

As to note, the incidence of neonatal seizure in our unit was reported rather higher than other units. This may be due to our frequent use of cerebral function monitoring (CFM, Natus Medical Inc., Seattle, WA) which was used to determine seizures from "jitteriness" and other non-epileptic paroxysmal movements even after seizures were clinically diagnosed by experienced neonatology staff.

We are aware of some limitations to our study: (i) The retrospective study design might not be the proper way to confirm examined relationships; (ii) the relatively small sample size of the study groups; (iii) many clinical conditions co-mingle originating from prematurity itself. In addition to lower gestational age, birth weight and other reported risk factors, worse respiratory function associated with massive pulmonary hemorrhage which may require more oxygen and mechanical ventilation in VLBWI may pose to the development of severe ROP. However, further studies in large population are warranted to clarify the influence of pulmonary hemorrhage as a risk factor for developing severe ROP.

\section{REFERENCES}

1. Maheshwari R, Kumar H, Paul VK, Singh M, Deorari AK, Tiwari HK. Incidence and risk factors of retinopathy of prematurity in a tertiary care newborn unit in New Delhi. Natl Med J India 1996; 9: 211-214.

2. Flynn JT, Bancalari E, Bawol R, et al. Retinopathy of prematurity. A randomized, prospective trial of transcutaneous oxygen monitoring. Ophthalmology 1987; 94: 630-638.

3. Repka MX, Hardy RJ, Phelps DL, Summers CG. Surfactant prophylaxis and retinopathy of prematurity. Arch Ophthalmol 1993; 111: 618-620.

4. Kim TI, Sohn J, Pi SY, Yoon YH. Postnatal risk factors of retinopathy of prematurity. prolonged use of ventilators. Paediatr Perinat Epidemiol 2004; 18: 130-134.

5. Gotsch F, Romero R, Kusanovic JP, et al. Preeclampsia and small-for-gestational age are associated with decreased concentrations of a factor involved in angiogenesis: soluble Tie-2. J Matern Fetal Neonatal Med 2008; 21: 389-402.

6. Lang DM, Blackledge J, Arnold RW. Is Pacific race a retinopathy of prematurity risk factor? Arch Pediatr Adolesc Med 2005; 159: 771-773.

7. Vinekar A, Dogra MR, Sangtam T, Narang A, Gupta A. Retinopathy of prematurity in Asian Indian babies weighing greater than 1250 grams at birth: ten year data from a tertiary care center in a developing country. Indian J Ophthalmol 2007; 55: 331-336.

8. Mayet I, Cockinos C. Retinopathy of prematurity in South Africans at a tertiary hospital: a prospective study. Eye (Lond) 2006; 20: 29-31.

9. Phan $\mathrm{MH}$, Nguyen PN, Reynolds JD. Incidence and severity of retinopathy of prematurity in Vietnam, a developing middle-income country. J Pediatr Ophthalmol Strabismus 2003; 40: 208-212.

10. Holmström G, van Wijngaarden P, Coster DJ, Williams KA. Genetic susceptibility to retinopathy of prematurity: the evidence from clinical and experimental animal studies. Br J Ophthalmol 2007; 91: 1704-1708.

11. Bizzarro MJ, Hussain N, Jonsson B, et al. Genetic susceptibility to retinopathy of prematurity. Pediatrics 2006; 118: 1858-1863.

12. International Committee for the Classification of Retinopathy of Prematurity. The International Classification of Retinopathy of Prematurity revisited. Arch Ophthalmol 2005; 123: 991-999. 
13. Papile L, Burstein J, Burstein R, Koffier A. Incidence and evolution of subependymal and intraventricular hemorrhage in premature infants: a study of infants < 1500gms. J Pediatr 1978; 92: 529-534.

14. Thomas K, Shah PS, Canning R, Harrison A, Lee SK, Dow KE. Retinopathy of prematurity: Risk factors and variability in Canadian neonatal intensive care units. J Neonatal Perinatal Med 2015; 8: 207-214.

15. Palmer EA, Flynn JT, Hardy RJ, et al. Incidence and early course of retinopathy of prematurity. The Cryotherapy for Retinopathy of Prematurity Cooperative Group. Ophthalmology 1991; 98: 1628-1640.

16. Lang DM, Blackledge J, Arnold RW. Is Pacific race a retinopathy of prematurity risk factor? Arch Pediatr Adolesc Med 2005; 159: 771-773.

17. Avery GB, Glass P. Retinopathy of prematurity: progress report. Pediatr Ann 1988; 17: 520: 532-533.
18. Glass P, Avery GB, Subramanian KN, Keys MP, Sostek AM, Friendly DS. Effect of bright light in the hospital nursery on the incidence of retinopathy of prematurity. N Engl J Med 1985; 313: 401-404.

19. Brooks SE, Marcus DM, Gillis D, Pirie E, Johnson MH, Bhatia J. The effect of blood transfusion protocol on retinopathy of prematurity: A prospective, randomized study. Pediatrics 1999; 104(3 Pt 1): 514-518.

20. Smolkin T, Steinberg M, Sujov P, Mezer E, Tamir A Makhoul IR. Late postnatal systemic steroids predispose to retinopathy of prematurity in very-low-birth-weight infants: a comparative study. Acta Paediatr 2008; 97: 322-326.

21. Evans N, Moorcraft J. Effect of patency of the ductus arteriosus on blood pressure in very preterm infants. Arch Dis Child 1992; 67(10 Spec No): 1169-1173. 